www.jmscr.igmpublication.org

Index Copernicus Value: 79.54

ISSN (e)-2347-176x ISSN (p) 2455-0450

crossrefDOI: https://dx.doi.org/10.18535/jmscr/v7i1.202

Original Research Article

\title{
GeneXpert or CBNAAT Based MTB Detection, Assay of Rifampicin Resistance and Comparison with Conventional Methods of Diagnosis
}

\author{
Authors \\ Sunil Kumar ${ }^{1}$, Chandan Kumar ${ }^{2 *}$, H.L. Mahto ${ }^{3}$ \\ ${ }^{1,2}$ Tutor, Department of Microbiology, Nalanda Medical College, Patna \\ ${ }^{3}$ Professor\& Head, Department of Microbiology, Nalanda Medical College, Patna \\ *Corresponding Author \\ Chandan Kumar
}

Tutor, Department of Microbiology, Nalanda Medical College, Kankarbagh main Road, near Bhutnath

Road, Patna-800026, India

Email: doctchandan@gmail.com, Mobile-7549025749

\begin{abstract}
Introduction: GeneXpert is a Cartridge based DNA amplification test. It has high sensitivity and specificity in comparison to conventional methods of diagnosis like $Z N$ staining and culture of Mycobacterium. It is also very rapid in comparison to conventional methods and in single setting MTB detection as well as Rifampicin resistance can be detected through this GeneXpert technique.

Material and Methods: Pulmonary and extra pulmonary samples as sputum, BAL, bronchoscopic aspirate, ascetic fluid, CSF, pleural effusion, are used. MTB detection is done with GeneXpert/MTB/RIF assay technique. ZN stain, LJ culture is done with the sample and comparison done with GeneXpert.

Results: Total 786 pulmonary and extra pulmonary samples are studied with GeneXpert technique. In which 520 pulmonary and 166 extra pulmonary samples are present. MTB detected in pulmonary samples are $101(19.4 \%)$, in which $18(n=101)$ are Rifampicin resistant. In extra pulmonary samples among 166 samples $26(15.6 \%)$ are MTB positive and 03(n=26) are Rifampicin resistant. Sensitivity and specificity both are high in GeneXpert than conventional methods of diagnosis like ZN staining and LJ culture.

Discussion and Conclusion: GeneXpert technique is rapid, sensitive, and very specific. In pulmonary and extra pulmonary cases specificity is almost $100 \%$ and sensitivity is also very high. Its diagnostic importance is increased many fold in smear negative pulmonary TB cases and extra pulmonary TB cases. Rifampicin resistance along with MTB detection can also be done with GeneXpert technique in single setting. The duration of time is also about 2 hour which is very short time period in comparison to conventional methods like Mycobacterium culture.
\end{abstract}

\section{Introduction}

Mycobacterium tuberculosis is a leading cause of death even in today's scenario in developing countries like India. In India over 2 lakhs deaths are reported every year due to tuberculosis. A total of 786 samples of suspected pulmonary and extra pulmonary tuberculosis were evaluated in this study. A/c to WHO, estimated incidence of TB cases in India in 2011 is about 2.2 million, while global incidence is 9.6 million cases ${ }^{1}$. Due to 
alarming rise in global incidence of TB as well as development of drug resistance, it is needed to develop a rapid diagnostic technique for the diagnosis of tuberculosis ${ }^{2}$. Tuberculosis is a major health problem in world with estimated new cases and death, every year is 8.7 million and 1.4 million respectively ${ }^{3}$.

According to WHO, India has the highest no. of TB cases and highest TB burden in the world ${ }^{4}$ The available techniques for the diagnosis of Tuberculosis is time taking and also has variable sensitivity and specificity. It leads to increased mortality and morbidity and development of drug resistance (MDR, XDR) TB patients ${ }^{5,6}$. Culture is the gold standard technique for the diagnosis of tuberculosis, but it is a slow process and it may take 2-8 weeks for the positive reports. Besides this Mycobacterium culture is a cumbersome procedure and it requires BSL II/III that is not affordable in everywhere ${ }^{7,8}$ Microscopic examination of smear for AFB (Acid Fast Bacilli) is fast method but its sensitivity is poor. Thus a rapid technique is needed for early, improved, sensitive and more effective diagnosis of tuberculosis which is based on the nucleic acid amplification technique ${ }^{9}$ Now a day's this rapid diagnostic technique is increasingly being used due to its rapid diagnosis and higher sensitivity and specificity ${ }^{10}$. Besides this through GeneXpert diagnostic method, sensitivity of Rifampicin can also be detected in same setting within few hours. Therefore now WHO has recommended the implementation of GeneXpert in national tuberculosis programme in developing countries. ${ }^{11}$ The GeneXpert MTB/RIF assay is a modern diagnostic technique which is automated, easy to operate and based on real time PCR analysis, perform sample processing and rapid diagnosis of RIF resistance in single setting in clinical specimen $^{12,13}$. In this CBNAAT/GeneXpert MTB/RIF technique result can be obtained within 2 hours ${ }^{3}$. This technique is not influenced by cross contamination and it requires minimum Biosafety facility and has high sensitivity and specificity even in smear negative pulmonary TB and extra pulmonary $\mathrm{TB} .^{910,14,15,16}$ In extra pulmonary TB its significance is also very important because it doesn't require multiple samples to be collected like in other methods of diagnosis. Usually in EPTB samples are collected by the invasive procedure and its difficult to obtain multiple samples, thus in such cases GeneXpert is a very useful tool

\section{Aims and Objectives}

The main objective of this study is to evaluate the sensitivity and specificity of Xpert/MTB/RIF assay for M.tuberculosis in pulmonary and extra pulmonary samples. The incidence of Rifampicin resistance among diagnosed $\mathrm{M}$. tuberculosis cases.

\section{Material and Methods}

Present study is done in the department of Microbiology, Nalanda Medical College \& Hospital, Patna between the period of June 2018 to January 2019. Total no. of 786 samples were processed and tested through GeneXpert Dx System(Cepheid). In this study samples of suspected pulmonary and extra pulmonary TB patients are received at the deprtment of Microbiology from the department of Medicine, Paediatrics and Surgery. Samples are received from both indoor as well as outpatient department. Among total of 786 samples, 620 were pulmonary specimen (sputum, BAL, bronchoscopic aspirate) and 166 were extra pulmonary specimen (pleural effusion, ascetic fluid, CSF, pus, pericardial fluid). The pulmonary samples- first decontaminated then smears are prepared and stained with ZN (ZiehlNeelsen) stain is done according to WHO protocol $^{17}$ 'GeneXpert MTB/RIF assay or CBNAAT is done through integrated diagnostic device, which perform sample processing and real time PCR analysis in a single step. The GeneXpert MTB/RIF assay consists of two components, a) plastic cartridge that contains PCR buffer and liquid sample processing and real-time PCR reagents which is in lyophilized form. b) The proper GeneXpert instrument, which controls intracartridge fluids and perform real-time PCR 
processing ${ }^{18}$. Real-time PCR processing is done according to given manual. The sample reagent and unprocessed sputum is mixed in a ratio of $2: 1$ in a $15 \mathrm{ml}$ Falcon tube. The tube is agitated twice and incubated for 15 minutes at room temperature. Now the $2 \mathrm{ml}$ of this mixed sample was introduced in the test cartridge by a sterile disposable pipette. Now this cartridge is loaded into GeneXpert instrument for the real-time PCR processing. The data interpretation of result is dependent on software of the equipment; it doesn't depend on the user. ${ }^{19}$. At last Culture is done on LJ (Lowenstein-Jensen) solid media by following the standard protocol.

The Extra pulmonary samples- first concentrated by centrifugation technique. Cytocentrifugation is done 3000rpm for 15 to 20 minutes. Again the sediments are used for the same process i.e $\mathrm{ZN}$ staining, GeneXpert/MTB/RIF test and culture is done on LJ media. Sensitivity and Specificity of each test is obtained by standard formulae.

\section{Results}

Out of total 786 samples, 520(66.2\%) are pulmonary specimen and $166(21.1 \%)$ are extra pulmonary. MTB detected through GeneXpert/ MTB/RIF assay is 101(19.4\%) in pulmonary specimen and $31(18.6 \%)$ in extra pulmonary specimen. Other conventional technique shows the following pattern, out of 520 pulmonary samples $75(14.4 \%)$ are culture positive on LJ media and $38(7.3 \%)$ samples are positive on $\mathrm{ZN}$ staining. Results seen among extra pulmonary samples, out of 166 EPTB samples 15(9.04\%) are culture positive and $08(4.8 \%)$ are positive on $\mathrm{ZN}$ staining. Besides this in pulmonary samples out of 101 positive specimens, $18(17.8 \%)$ are Rifampicin resistant and out of 26 positive extra pulmonary samples 01(3.8\%) are Rifampicin resistant.

Table-I Results of GeneXpert assay

\begin{tabular}{|l|c|c|c|}
\hline Results & Pulmonary samples & Extra pulmonary. samples & Total \\
\hline No. of samples & 520 & 166 & 786 \\
\hline MTB detected & $101(19.4 \%)$ & $26(15.6 \%)$ & 127 \\
\hline Rif. Resistance detected & $18(17.4 \%), \mathrm{n}=101$ & $03(11.5 \%), \mathrm{n}=26$ & 21 \\
\hline
\end{tabular}

Table-II Comparison between GeneXpert, culture and ZN staining results

\begin{tabular}{|l|c|c|c|}
\hline Sample types & GeneXpert (positive) & Culture (positive) & ZN stain (positive) \\
\hline Pulm. Samples, $(\mathrm{n}=520)$ & $101(19.4 \%)$ & $90(17.3 \%)$ & $38(7.3 \%)$ \\
\hline EPTB Samples, $(\mathrm{n}=166)$ & $26(15.6 \%)$ & $15(9.0 \%)$ & $05(3.0 \%)$ \\
\hline Total, $(786)$ & 127 & 105 & 43 \\
\hline
\end{tabular}

The above comparative study shows that sensitivity of GeneXpert is highest among all these three methods. GeneXpert has detected MTB in all the culture positive samples $(n=90)$ and 11 more samples are detected positive for
MTB that are culture negative. Similarly all the smear positive samples i.e $\mathrm{ZN}$ stain positive samples are detected MTB positive by GeneXpert and 63 smear negative samples are also detected positive by GeneXpert technique.

Table-III Comparison of ZN stain positive smear with LJ culture and GeneXpert method

\begin{tabular}{|l|c|c|c|c|}
\hline \multicolumn{2}{|c|}{ Result of LJ } & culture and & GeneXpert \\
\hline Smeare result & $\begin{array}{c}\text { LJ culturenegative, } \\
\text { GeneXpert -ve }\end{array}$ & $\begin{array}{c}\text { LJ culture negative, } \\
\text { GeneXpert positive }\end{array}$ & $\begin{array}{c}\text { LJ culture positive, } \\
\text { GeneXpert positive }\end{array}$ & Total \\
\hline ZN smear positive & 0 & 0 & 43 & 43 \\
\hline ZN smear negative & 659 & 22 & 62 & 742 \\
\hline Total & 659 & 22 & 105 & 786 \\
\hline
\end{tabular}

The above table shows that all smear positive (ZN stainpositive) samples are positive for LJ culture and GeneXpert. Many smear negative $(\mathrm{ZN}-\mathrm{ve})$ samples are culture positive and all culture positive samples are also positive by GeneXpert. 


\section{JMSCR Vol||07||Issue||01||Page 1150-1156||January}

Rifampicin resistance among MTB positive samples

\begin{tabular}{|l|c|c|}
\hline Sample & GeneXpert positive MTB & Rifampicin resistant \\
\hline Pulmonary, $(\mathrm{n}=520)$ & 101 & 18 \\
\hline Extra pulmonart, $\mathrm{n}=166$ & 26 & 03 \\
\hline
\end{tabular}
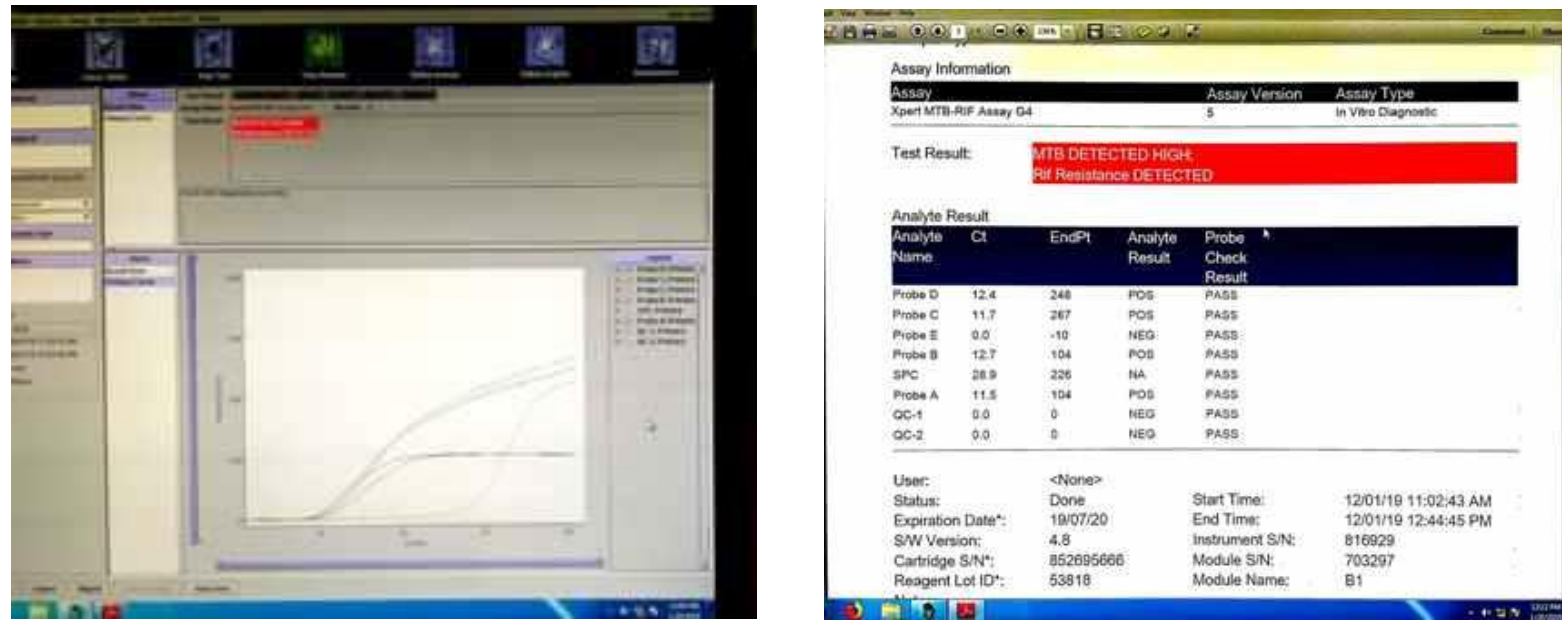

GeneXpert/MTB/RIF assay detecting MTB positive and Rifampicin resistance
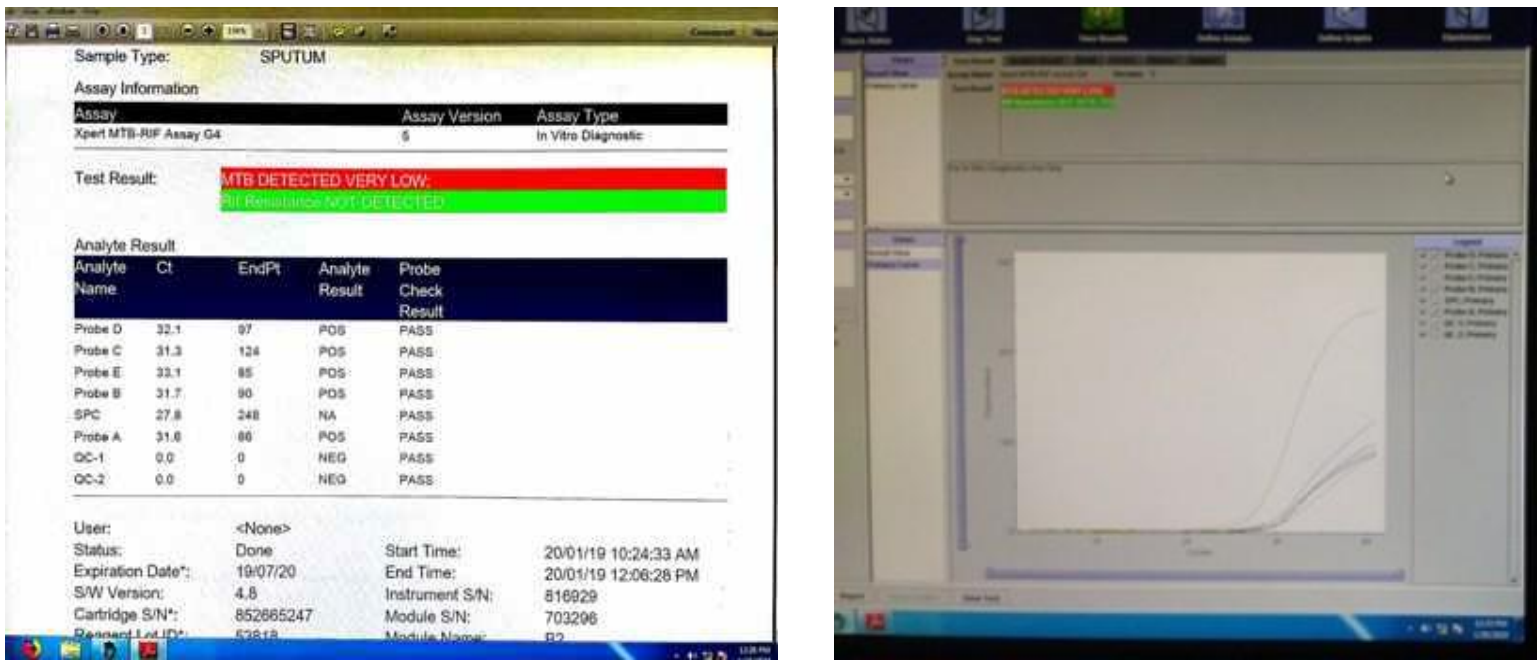

GeneXpert/MTB/RIF assay detecting MTB positive without Rifampicin resistance

Out of 101 GeneXpert positive pulmonary samples for MTB, 18 are Rifampicin resistant and among 26 extra pulmonary samples 03 are resistant for Rifampicin.

\section{Discussion}

$\mathrm{ZN}$ staining is a conventional method for diagnosis of MTB from clinical specimen and its sensitivity is less than culture of Mycobacterium. For $\mathrm{ZN}$ staining to be positive large bacillary load $10^{5}$ mycobacterium $/ \mathrm{ml}$ of sample is required ${ }^{20}$. The conventional culture is time consuming and it requires Bio safety laboratory and trained persons. GeneXpert/MTB/RIF assay is a rapid nucleic acid amplification based test and it requires less technical expertise. Other advantage is that in a single setting the MTB detection as well as Rifampicin resistance can be detected through GeneXpert technique. Through GeneXpert technique diagnosis can be done within 2 hour. With the help of GeneXpert pulmonary as well as extra pulmonary MTB can be diagnosed by same process.

In this study we have compared the efficacy of GeneXpert MTB/RIF assay with ZN staining and $\mathrm{LJ}$ culture results for both pulmonary and extra pulmonary specimen. Among 786 pulmonary and extra pulmonary, samples $127(16.2 \%)$ are positive 
for MTB by GeneXpert/RIF assay. Among this 127 samples $43(5.4 \%)$ samples are smear positive ie $\mathrm{ZN}$ stain positive, it means $84(10.7 \%)$ smear ve samples are also diagnosed positive by GeneXpert technique. It means $10.4 \%$ more samples are diagnosed positive by GeneXpert technique than $\mathrm{ZN}$ staining. Similar results are obtained by Batz et al in their study who found greater detection rate of MTB with GeneXpert than culture and $\mathrm{ZN}$ staining ${ }^{21}$. In this study GeneXpert $95 \%$ smear positive and culture positive samples are detected positive, but in our study $100 \%$ are detected positive by GeneXpert. Zeka et al also reported the similar kind of result i.e $100 \%$ of culture and smear positive samples are positive by GeneXpert similar to our study ${ }^{15}$.

\section{GeneXpert result compared with LJ culture and Smear positive pulmonary samples.}

\begin{tabular}{|c|c|c|c|c|c|}
\hline & \multicolumn{3}{|c|}{ smear positive } & \multicolumn{2}{|c|}{ Total } \\
\hline & $\begin{array}{c}\text { culture } \\
\text { positive }\end{array}$ & culturenegative & $\begin{array}{c}\text { culture } \\
\text { positive }\end{array}$ & culture -ve & \\
\hline GeneXpertpositive & 38 & 0 & 52 & 11 & 101 \\
\hline GeneXpertnegative & 0 & 0 & 0 & 419 & 419 \\
\hline Total & 38 & & 52 & 430 & 520 \\
\hline Sensitivity & & 100 & & & \\
\hline Specificity & & 100 & & & \\
\hline
\end{tabular}

In the above mentioned chart we have compared the sensitivity and specificity of the GeneXpert with smear -ve and smear positive specimen. We have found that in smear positive samples sensitivity and specificity of the GeneXpert is $100 \%$ and $100 \%$ respectively. While in smear negative samples sensitivity and specificity of GeneXpert is $82.5 \%$ and $100 \%$ respectively. Similar results are found in the study of Zeka et $a l^{15}$ for smear positive samples the sensitivity and specificity is $100 \%$ while in smear -ve cases sensitivity is about $68 \%$ and specificity is of $100 \%$. Boehm et al. in their study found similar result with smear positive samples while for smear -ve samples sensitivity is of $77 \%^{19}$. In case of extra pulmonary samples, in our study GeneXpert has detected $12.6 \%$ more positive cases than $\mathrm{ZN}$ stain (smear positive) and $6.6 \%$ more positive cases than LJ culture. It indicates higher sensitivity of GeneXpert than ZN staining and LJ culture. Similar results have been found in study of HIllemann et $a l^{10}$. In the study of Tortoli et $a l^{22}$. similar kind of results have been found as compare to our study i.e sensitivity and specificity is $86.9 \%$ and $99.7 \%$ respectively in EPTB.

Therefore it is very clear from our study that in both pulmonary TB as well as EPTB cases sensitivity and specificity of GeneXpert testing is very high. The time spends for the diagnosis of MTB and Rifampicin resistance is of about 2 hour which is very fast. Hence management of TB patients and prevention of MDR TB has increased tremendously with the help of GeneXpert technique.

\section{Conclusion}

GeneXpert technique is rapid, sensitive and specific test. It has also very important role in detection of Rifampicin resistance. Its sensitivity and specificity is also high in EPTB specimen. In our study, case detection rate of GeneXpert in pulmonary cases $12.1 \%$ and $2.1 \%$ more than $\mathrm{ZN}$ staining and LJ culture results respectively. In EPTB case detection rate is $12.6 \%$ and $6.6 \%$ more than $\mathrm{ZN}$ staining and $\mathrm{LJ}$ culture respectively. Hence in pulmonary, extra pulmonary, smear positive and smear negative in all cases sensitivity and specificity is higher than conventional technique.

\section{Bibliography}

1. TB Stastics for India. (2012). TB Facts. Retrieved April 3, 2013, from http://wwwltbfcts.org/tb-statisticsindia.html 
2. Helb D, Jones M, Story E, Boehme C, Wallace E, Ho K, Kop J, et al. Rapid detection of Mycobacterium tuberculosis and rifampin resistance by use of ondemand, near-patient technology. J Clin Microbiol. 2010;48:229-237. [PMC free article] [PubMed]).

3. World Health Organization. Executive Summary, Global Tuberculosis Report 2012. Available at: http://www.who.int/tb/publications/global _report/gtbr12_executivesummary.pdf

4. WHO. Guidelines for the programmatic management of drug-resistant tuberculosis. Geneva: World Health Organization; 2016.)

5. Farmer, P., J. Bayona, M. Becerra, J. Furin, C. Henry, H. Hiatt, J. Y. Kim, C. Mitnick, E. Nardell, and S. Shin. 1998. The dilemma of MDR-TB in the global era. Int. J. Tuberc. Lung Dis. 2:869-876. PubMed Web of Science Google Scholar)

6. Moore, D. A., C. A. Evans, R. H. Gilman, L. Caviedes, J. Coronel, A. Vivar, E. Sanchez, Y. Pinedo, J. C. Saravia, C. Salazar, R. Oberhelman, M. G. HollmDelgado, D. LaChira, A. R. Escombe, and J. S. Friedland. 2006. Microscopic observation drug-susceptibility assay for the diagnosis of TB. N. Engl. J. Med. 355:1539-1550. CrossRef PubMed Web of Science Google Scholar.

7. Alvarez-Uria G, Azcona JM, Midde M, Naik PK, Reddy S, Reddy R. Rapid diagnosis of pulmonary and extra pulmonary tuberculosis in HIV-infected patients Comparison of LED fluorescent microscopy and the geneXpert MTB/RIF assay in a district hospital in India. Tuber Res Treatment. 2012;2012:1-4.[PMC free article] [PubMed]),

8. Boehme CC, Nabeta P, Hillemann D, Nicol MP, Shenai S, Krapp F, et al. Rapid molecular detection of tuberculosis and rifampin resistance. $\mathrm{N}$ Eng $\mathrm{J}$ Med.
2010;363(11):1005-1015. [PMC free article] [PubMed]

9. Anonymous 2009. Updated guidelines for the use of nucleic acid amplification tests in the diagnosis of tuberculosis. MMWR Morb. Mortal. Wkly. Rep. 58:7-10. PubMed Google Scholar)

10. Hillemann D, Rüsch-Gerdes S, Boehme C, Richter E. Rapid molecular detection of extra pulmonary tuberculosis by the automated GeneXpert MTB/RIF system. J Clin Microbio. 2011;49(4):1202-1205. [PMC free article] [PubMed])

11. World Health Organization, Rapid Implementation of the Xpert MTB/RIF Diagnostic Test, World Health Organization. 2011. Available at: http://whqlibdoc.who.int/publications/201 1/9789241501569_eng.pdf.)

12. Blakemore R., et al. 2010. Evaluation of the analytical performance of the Xpert MTB/RIF assay. J. Cin Microbiol. 48:2495-2501. Google Scholar.

13. Helb D., et al. 2010. Rapid detection of Mycobacterium tuberculosis and rifampin resistance by use of on-demand, nearpatient technology. J. Clin. Microbiol. 48:229-237. Abstract/FREE Full Text Google Scholar)

14. Armand S, Vanhuls P, Delcroix G, Courcol R, Lemaitre N. Comparison of the Xpert MTB/RIF test with an IS6110TaqMan real-time PCR assay for direct detection of Mycobacterium tuberculosis in respiratory and nonrespiratory specimens. J Clin Microbiol. 2011;49:1772-1776. [PMC free article] [PubMed])

15. Zeka AN, Tasbakan S, Cavusoglu C. Evaluation of the GeneXpert MTB/RIF assay for rapid diagnosis of tuberculosis and detection of rifampin resistance in pulmonary and extrapulmonary specimens. J Clin Microbiol. 2011;49(12):4138-4141. [PMC free article] [PubMed], 
16. Vadwai V, Boehme C, Nabeta P, Shetty A, Alland D, Rodrigues C. Xpert MTB/RIF: a new pillar in diagnosis of extrapulmonary tuberculosis? J Clin Microbiol. 2011;49(7):2540-2545. [PMC free article] [PubMed]

17. International Union against Tuberculosis and Lung Disease. Sputum examination for tuberculosis by direct microscopy in low income countries. Technical Guide. 5th ed. Paris, France: IUATLD; 2000.)

18. Raja, S., J. Ching, L. Xi, S. J. Hughes, R. Chang, W. Wong, W. McMillan, W. E. Gooding, K. S. McCarty, Jr., M. Chestney, J. D. Luketich, and T. E. Godfrey. 2005. Technology for automated, rapid, and quantitative PCR or reverse transcriptionPCR clinical testing. Clin. Chem. 51:882890. Abstract/FREE Full Text Google Scholar)

19. Boehme CC, Nabeta P, Hillemann D, Nicol MP, Shenai S, Krapp F, et al. Rapid molecular detection of tuberculosis and rifampin resistance. $\mathrm{N}$ Eng $\mathrm{J}$ Med. 2010;363(11):1005-1015. [PMC free article] [PubMed])

20. Lawn SD, Nicol MP. Xpert® MTB/RIF assay: development, evaluation and implementation of a new rapid molecular diagnostic for tuberculosis and rifampicin resistance. Fut Microbiol. 2011;6(9):10671082. [PMC free article] [PubMed].)

21. Batz HG, Cooke GS, Reid SD. Towards lab-free tuberculosis diagnosis. Treatment Action Group, the TB/HIV Working Group of the Stop TB Partnership, Imperial College, and the MSF Access Campaig. 2011.).

22. Tortoli E, Russo C, Piersimoni C, Mazzola E, Dal Monte $\mathrm{P}$, Pascarella $\mathrm{M}$, et al. Clinical validation of Xpert MTB/RIF for the diagnosis of extrapulmonary tuberculosis. Eur Respir J. 2012;40: 442447. [PubMed]. 